\title{
Surgical Treatment of a Popliteal Artery Pseudoaneurysm after Total Knee Arthroplasty
}

\section{B. Matías García*, A Sánchez Gollarte, F Ruiz Grande, S Barrena Blázquez, T Ratia Jiménez, RG Alvarado Hurtado and F Noguerales Fraguas}

School of Medicine, Rey Juan Carlos University, Móstoles, Spain

\begin{abstract}
Summary
Introduction: Vascular complications after total knee arthroplasty (TKA) are uncommon but they can compromise the viability of the limbs. The incidence of popliteal pseudoaneurysms after total knee arthroplasty varies from 0.03 to $0.5 \%$. We present the case of a popliteal pseudoaneurysm as a complication after a TKA.

Clinical case: A 76-year-old woman with a diagnosis of right knee osteoarthritis is undergoing TKA. During the postoperative period, the patient presented a pulsating and progressive growing mass in the popliteal gap, pain and functional impotence. Doppler ultrasound and computed tomography (CT) confirmed the presence of a pseudoaneurysm. A pseudo endoaneurysmorrhaphy was performed by posterior access.

Discussion: Popliteal pseudoaneurysm is a rare complication after TKA and many factors related to surgery are involved in its etiology. Typically, it appears as a painful pulsatile mass at the first fifteen days postoperatively. CT angiography is the standard for diagnosis and it also allows to plan the management. Treatment varies from surgical or endovascular approach to compression or ultrasound-guided thrombosis.
\end{abstract}

\section{Introduction}

Vascular complications after TKA are uncommon but, when appear, they can compromise the viability of the limbs. The most common vascular lesions include arterial thrombosis, arteriovenous fistula, direct or indirect vascular damage, and aneurysms and pseudoaneurysms [1-5].

The incidence of popliteal pseudoaneurysms after TKA varies from 0.03 to $0.5 \%$ depending on the series $[1,2,6-8]$. Clinically, they present as a pulsatile hemat- oma with swelling of the popliteal region [1,3-7,9-12]. Its presentation is similar to deep vein thrombosis, so the differential diagnosis is usually made after ultrasound $[6,10]$. Therefore, early diagnosis and treatment are important to prevent associated complications. We present the case of a popliteal pseudoaneurysm in a 76-year-old woman after a TKA.

\section{Clinical Case}

A 76-year-old woman with history of hypertension and right knee osteoarthritis underwent total knee arthroplasty that went without incident. On the seventh postoperative day, she presented edema of the lower limb. Physical examination revealed a pulsatile, painful and progressive growing mass in the popliteal gap, not erythematous and without temperature rise, associated with functional impotence due to compression of the surrounding structures. Distal pulses were palpable and the sensitivity was preserved. Doppler ultrasound was performed on the seventh postoperative day and showed a fusiform dilation of the popliteal artery with turbulent flow inside, suggesting a pseudoaneurysm. She was assessed by Vascular Surgery and was referred to consultations to complete the study on an outpatient basis. She was discharged on the ninth postoperative day. CT angiography on an outpatient basis confirmed the presence of a pseudoaneurysm of $5.5 \times 3.8 \times$ 5.9 centimetres in diameter (transverse $x$ anteroposterior $x$ craniocaudal diameters) (Figure 1).

Pseudo endoaneurysmorrhaphy by lateral suturing of the popliteal artery by posterior incision was performed electively in the following month: The sack 


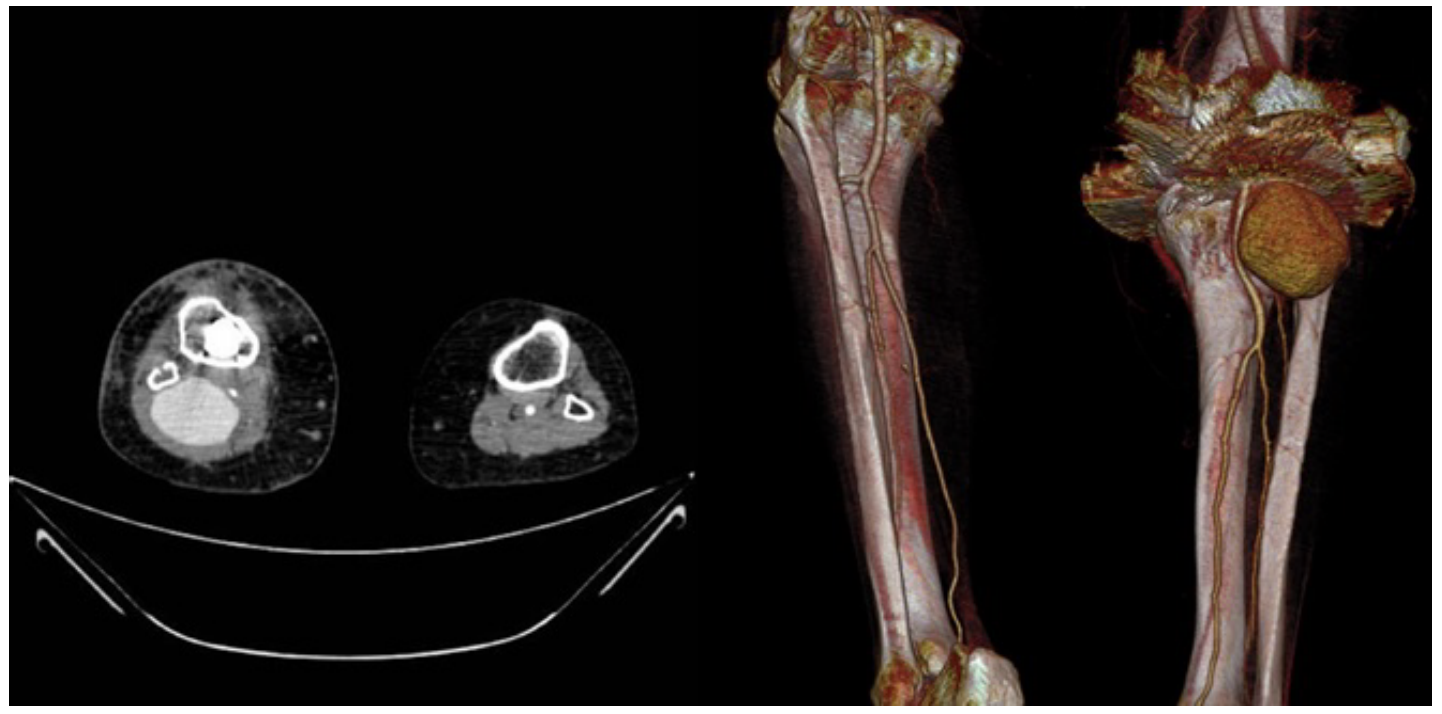

Figure 1: CT angiography image and 3D reconstruction showing the popliteal artery pseudoaneurysm.

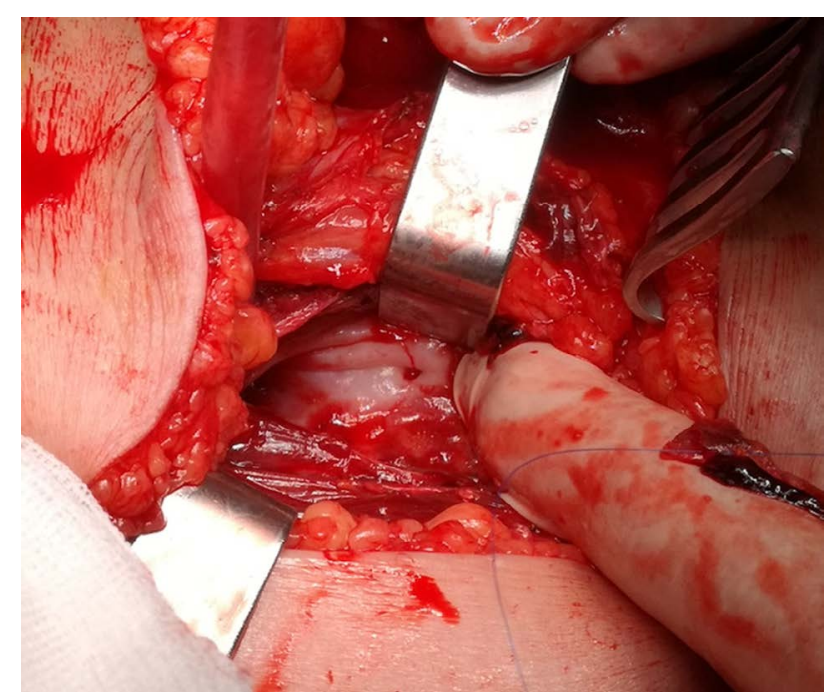

Figure 2: Identification of the bleeding point.

was opened posteriorly identifying the bleeding point (Figure 2). A lateral tear in the second portion of the popliteal artery was identified and repaired by a continuous lateral suture with monofilament 5/0 (Figure 3), verifying distal pulses. In the postoperative period, the patient evolved favourably with preserved distal pulses, improved symptomatology and there was no complication with the implant. She was discharged on the third postoperative day. The last review was 2 months after the intervention, the patient was asymptomatic, distal pulses were present and the ultrasound showed no recurrence data.

\section{Discussion}

Arterial complications after TKA are unusual, with an approximate incidence of $0.25 \%$ of all interventions. Popliteal artery is the most frequently affected artery due to its proximity to the joint. The incidence of popliteal pseudoaneurysm after a TKA varies from 0.03 to $0.5 \%[1,2,6-8]$. It is more frequent in revision than in

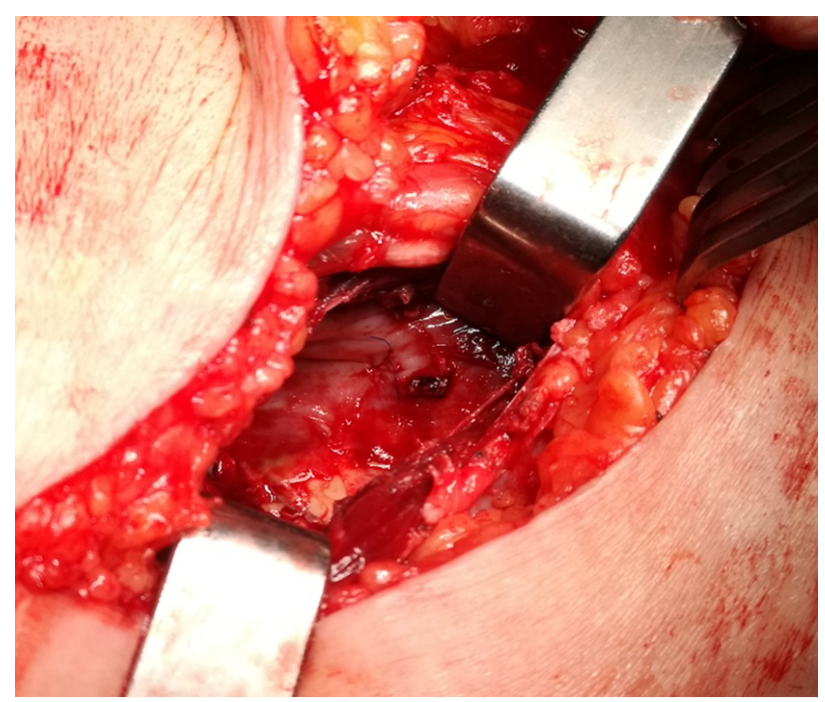

Figure 3: Repair by continuous lateral suture.

primary surgeries due to the existence of fibrosis that hinders dissection of tissues [6].

Symptoms are usually evident in the immediate postoperative period, around the first 15 days [6], although in up to $40 \%$ of cases the diagnosis is made delayed, between the first and fourth month after surgery $[1,7]$. Typically, it presents as pulsatile mass at popliteal fossa, associated with pain, bruising and/or edema, and sometimes paraesthesia due to the compression of peroneal nerve [1,3-7,9-11].

Due to its clinical presentation (lower limb edema, pain, etc.) and the history of recent surgery, popliteal pseudoaneurysm after TKA is often diagnosed after a Doppler ultrasound performed to dismiss deep vein thrombosis (DVT). However, CT-angiography is the gold standard and it is also useful to plan the therapeutic strategy [6].

Regarding the etiology of pseudoaneurysms after TKA, several mechanisms have been described. On 
the one hand, flexion movements during surgery can produce intimate lesions due to the proximity of the popliteal vessels to the knee joint. In the same way, the use of orthopaedic instruments (oscillating saw, retractors, etc.) as well as the surgical technique itself (resection of the femoral and tibial condyles and the release of the posterior capsule) have been implicated in the pathogenesis of pseudoaneurysms $[1,2,7]$. Finally, some authors have associated pre-existing vascular disease with this complication $[3,4,6,7]$. In our case, the exact mechanism by which the popliteal artery was damaged is unknown, although the arterial injury is suspected to be produced during the tibial cut.

In relation to the treatment of popliteal pseudoaneurysms, there are several therapeutic strategies. Open surgery by vein bypass has been widely used with excellent long-term results [8]. However, it is hampered by the presence of fibrosis and anatomical changes due to previous surgery, which increase the risk of injury of adjacent structures and prosthesis infection [1]. Endovascular treatment with stents has been tried in the management of popliteal pseudoaneurysms with good results. However, the presence of a radiopaque prothesis increases the number of projections required to identify the lesion. On the other hand, as knee joint present a large range of movement, the placement of a stent in this area can lead to its breakage or migration [1]. Published results using stents are satisfactory, although the lack of long-term follow-up does not allow to generalize their indication [2].

Less invasive therapeutic alternatives are ultrasound-guided compression or thrombin injection in order to achieve thrombosis [1]. The latter has good results, but few cases has been reported and data are not still conclusive [2].

Although this technique is described in the literature, there is not much experience and its use is not standardized. In the case of large pseudoaneurysms such as ours, we prefer the use of open surgery over endovascular treatment. With respect to bypass, we prefer pseudo endoaneurysmorraphy due to a reduced need for tissue dissection since it is a region undergoing previous surgery and therefore presents an increased risk of injury. That together with the added risk of infection and other complications associated with the placement of a prosthesis, makes us contemplate pseudo endoaneurysmorraphy as the best option for the treatment of popliteal pseudoaneumysm after TKA.

\section{Conclusion}

Popliteal pseudoaneumysm are an uncommon complication after an ATR and need to make a differential diagnosis with deep vein thrombosis. In terms of management, pseudo endoaneurysmorraphy seems to be a decisive and safe option in patients undergoing pri- or surgery as in our case, with good short-term results. However, studies comparing long-term results with other treatments are necessary.

\section{References}

1. Blanco Cañibano E, García Fresnillo B, Guerra Requena M (2008) Pseudoaneurysm of the popliteal artery as a complication of knee prosthesis surgery: Endovascular treatment. Angiología 60: 49-54.

2. López Arquillo I, Gallego Ferreiroa C, Fraga Muñoz E, Vidal Rey J, Bolívar Gómez T, et al. (2011) Poptileal artery complications after total knee arthroplasty. Angiology 63: 250-253.

3. Geertsema D, Defoort K, Van Hellemondt G (2012) Popliteal Pseudoaneurysm after total knee arthroplasty: A report of 3 cases. The journal of Arthroplasty 27: 1581e1-1581e4.

4. Butt U, Samuel R, Sahu A, Johnson D (2010) Arterial injury in total knee arthroplasty. The Journal of Arthroplasty 8: 1311-1318.

5. Sandoval E, Ortega F, García-Rayo M, Resines C (2008) Popliteal pseudoaneurysm after total knee arthroplasty secondary to intraoperative arterial injury with a surgical pin: review of the literature. J Arthroplasty 23: 1239.e71239.e11.

6. Ammori M, Evans A, Mclain A (2016) Popliteal artery pseudoaneurusm after total knee arthroplasty. The journal of Arthroplasty 31: 2004-2007.

7. González Rodríguez J, Jordan Sales M, Aguilera Roig X, Monllau García J, Celaya Ibáñez F (2012) Pseudoaneurisma poplíteo como complicación de la artroplastia total de rodilla. Revisión y actualización bibliográfica a propósito de un caso. Revista Española de Cirugía Ortopédica y Traumatología 56: 205-209.

8. Troutman D, Dougherty M, Spivack A, Calligaro K (2013) Updated strategies to treat acute arterial complications associated with total knee and hip arthropasty. J Vasc Surg 58: 1037-1042.

9. Karaca O, Akpinar E, Omanygenic O, Cakal B, Boztosun B (2015) Endovascular repair of a popliteal artery pseudoaneurysm following total knee arthroplasty. J Clin Diagn Res 9: 1.

10. Tejero-García S, Lirola Criado JF, Ast MP, Fernández de Bobadilla GD (2014) Popliteal pseudoaneurysm after unicompartimental knee replacement: A case report. Knee 21: $597-599$

11. Shin Y, Hwang Y, Savale A, Han S (2014) Popliteal artery pseudoaneurysm following primary total knee arthroplasty. Knee Surg Relat Res 26: 117-120.

12. Plagnol P, Dinard N, Bruneteau P, Roncheau V (2001) Pseudoaneurysm of popliteal artery complicating a total knee replacement: A successful percutaneous endovascular treatment. Eur J Vasc Endovasc Surg 21: 81-83. 